\title{
RESPOSTAS FISIOLÓGICAS AO EXERCÍCIO AQUÁTICO EM GESTANTES HIPERTENSA E NORMOTENSA: RELATO DE CASO CONTROLE
}

\author{
Guilherme Rosa ${ }^{1,3}$, Ighor Henriques ${ }^{1,3}$, Ingrid de Freitas Soutinho ${ }^{2}$, Gustavo Neves \\ Monteiro da Silva ${ }^{2}$, Danielli Mello ${ }^{1,4}$, Fábio Dutra Pereira ${ }^{2}$ \\ ${ }^{2}$ Grupo de Pesquisas em Exercício Físico e Promoção da Saúde - UCB/RJ. \\ ${ }^{2}$ InFocus Research Group - UCB/RJ. \\ ${ }^{3}$ Faculdade Bezerra de Araújo - FABA/RJ. \\ ${ }^{4}$ Escola de Educação Física do Exército - EsEFEx/RJ.
}

OPEN ACCES

Correspondencia Professor Guilherme Rosa Grupo de Pesquisas em Exercício Físico Promoção da Saúde - UCB/RJ. Departamento de Educação Física. Av. Santa Cruz, 1631. CEP: 21710-250. Rio de Janeiro-RJ. Brasil. (55) 21 3216-7700 grfitness@hotmail.com

Funciones de los autores: Todos los autores trabajaro equitativamente en la consecución del trabajo.

Recibido: 23/05/2018 Aceptado: $12 / 11 / 201$ Publicado: 31/01/2019

Citación:

Rosa, G., Henriques, I., Freitas-Soutinho I., Monteiro-Da Silva, G. N., Mello, D., \& Dutra-Pereira, F. (2019). Respostas fisiológicas ao exerício aquático em gestantes hipertensa e normotensa: relato de caso controle. RIAA. Revista de Investigación en Actividades Acuáticas, 3(5), 14-17 https://doi.org/10.21134/riaa.v3i5.1542

@creative (c) (1)(2) (2)

Creative Commons License Esta obra está bajo una licencia de Esta obra está bajo una licencia de NoComercial-Compartir-Igua 4.0 Internaciona
Contexto: Exercícios aquáticos são prescritos para gestantes devido seus benefícios para a mãe e bebê, entretanto, é importante conhecer as respostas fisiológicas à prática do exercício nessa população.

Objetivos: avaliar as adaptações fisiológicas ao exercício aquático em uma gestante hipertensa e uma gestante normotensa.

Método: Relato de caso/controle composto por 2 mulheres gestantes (1 hipertensa, 31 anos, 102,1kg, 1,60m e sedentária; e 1 normotensa, 35 anos, $97,8 \mathrm{~kg}, 1,73 \mathrm{~m}$, e fisicamente ativa), ambas no terceiro trimestre de gestação. Avaliou-se a frequência cardíaca (FC), pressão arterial sistólica (PAS), pressão arterial diastólica (PAD), duplo produto (DP), variabilidade da frequência cardíaca (VFC), e glicose sanguínea (GLI) em quatro momentos distintos: em decúbito dorsal após repouso de 15' (M1), em imersão em piscina com água na altura do manúbrio esternal após repouso de $5^{\prime}$ (M2), imediatamente após a sessão de exercício aquático (M3), e em decúbito dorsal após repouso de $5^{\prime}$ (M4) respectivamente, exceto para a GLI que foi aferida apenas em M1, M3 e M4. A sessão de exercício consistiu em movimentos de adução e abdução horizontal de ombros combinados com flexão e extensão de quadril sem a utilização de implementos e intensidade entre $60 \%$ e $80 \%$ da FC máxima.

Resultados: observou-se que as variáveis hemodinâmicas apresentaram modificações apenas em função da imersão na piscina. A sessão de exercício provocou elevação nas variáveis analisadas em ambas participantes, exceto para GLI que apresentou redução. Destaca-se a melhora em FC, PAS, PAD, DP, VFC e GLI na gestante hipertensa em M4 em comparação ao M1.

Conclusoes: o exercício aquático provocou adaptações fisiológicas positivas apresentando-se como uma possível modalidade a ser praticadas por gestantes.

Palavras-chave: exercício físico, hipotensão pós exercício, gestação, hemodinâmica, promoção da saúde.

\section{Titulo: Respuestas fisiológicas al ejercicio acuático en embarazadas normotensa y hipertensa: relato de caso control}

Antecedentes: El ejercício acuático se prescriben para las embarazadas debido a sus beneficios para la madre y el bebé, sin embargo, es importante conocer las respuestas fisiológicas a la práctica del ejercicio en esa población.

Objetivos: evaluar las adaptaciones fisiológicas al ejercicio acuático en una embarazada hipertensa y una embarazada normotensa.

Método: Relato de caso/control compuesto por 2 mujeres embarazadas (1 hipertensa, 31 años, 102,1kg, 1,60m y sedentária; y 1 normotensa, 35 años, $97,8 \mathrm{~kg}, 1,73 \mathrm{~m}$ y fisicamente activa), ambas en el tercero trimestre de embarazo. Fueron evaluadas la frecuencia cardiaca (FC), pressión arterial sistólica (PAS), pressión arterial diastólica (PAD), doble producto (DP), variabilidad de la frecuência cardaaca (VFC), y glucosa (GLU) en cuatro momentos distintos: en posición supina despues de reposo de $15^{\prime}$ (M1), en imersión en piscina con el agua en el manubrium esternal despues de reposo de 5' (M2), imediatamente despues de la sessión del ejercício acuático (M3), y en posición supina despues de reposo de 5' (M4) respectivamente, exceto para la GLU medida solamente en M1, M3 y M4. La sessión de ejercício consistió en movimientos de adución y abdición horizontal de ombros combinados con flexión y extensión de cadera sin ninguno implemento y intensidad entre $60 \%$ y $80 \%$ da la FC maxima.

Resultados: se observó que las variables hemodinámicas presentaron modificaciones sólo en función de la inmersión en la piscina. La sesión de ejercicio provocó elevación en las variables analizadas en ambas participantes, excepto para GLU que presentó reducción. Se destaca la mejora en FC, PAS, PAD, DP, VFC y GLI en la embarazada hipertensa en M4 en comparación con el M1.

Conclusiones: el ejercicio acuático provocó adaptaciones fisiológicas positivas presentándose como una posible modalidad a ser practicadas por mujeres embarazadas.

Palabras clave: ejercício físico, hipotensión post ejercício, embarazo, hemodinâmica, promoción de la salud.

Title: Physiological responses to aquatic exercise in normotensive and hypertensive pregnant women: case control report

Background: Aquatic exercises are prescribed for pregnant women because of their benefits to the mother and baby, however, it is important to know the physiological responses to exercise in this population.

Objectives: to evaluate the physiological adaptations to aquatic exercise in a hypertensive pregnant woman and a normotensive pregnant woman.

Method: A case-control study comprised of two pregnant women (1 hypertensive, 31 years old, $102.1 \mathrm{~kg}, 1.60 \mathrm{~m}$ and sedentary, and 1 normotensive, 35 years old, $97.8 \mathrm{~kg}, 1.73 \mathrm{~m}$, and physically active), both in the third trimester of pragnancy. Heart rate (HR), systolic blood pressure (SBP), diastolic blood pressure (DBP), double product (SD), heart rate variability (HRV) and blood glucose (GLI) were evaluated at four different times: dorsal decubitus after resting 15 '(M1), immersion in a pool with water at the height of sternal manubrium after 5 ' resting (M2), immediately after the aquatic exercise (M3), and in dorsal decubitus after $5^{\prime}$ resting (M4) respectively, except for the GLI that was measured only in M1, M3 and M4. The exercise session consisted of horizontal shoulder adduction and abduction movements combined with flexion and hip extension without any implements and intensity between $60 \%$ and $80 \%$ of maximal HR.

Results: it was observed that the hemodynamic variables presented modifications only as a function of immersion in the pool. The exercise session caused an increase in the variables analyzed in both participants, except for GLI that presented reduction. Its posible to highlight the improvement in HR, SBP, DBP, DBP, HRV and GLI in the hypertensive pregnant woman in M4 compared to M1.

Conclusions: the aquatic exercise caused positive physiological adaptations presenting as a possible modality to be practiced by pregnant women.

Key words: physical exercise, post exercise hypotension, pregnancy, hemodynamics, health promotion. 


\section{Introdução}

Nas últimas décadas, a prática de exercícios físicos entre as mulheres ganhou ênfase e, embora persistam controvérsias quanto à sua realização durante o período gestacional, essa prática vem crescendo nesse grupo. Em décadas passadas, as gestantes eram aconselhadas a reduzirem, ou até mesmo a interromperem, as suas atividades ocupacionais, acreditando-se que isso poderia melhorar os desfechos perinatais (Artal \& Gardin, 1999).

Alguns benefícios, como o controle do peso e o auxílio na prevenção de diabetes gestacional, têm sido apontados como secundários à realização de atividade física pela gestante (Haskell et al., 2007; Dye et al., 1997). As atividades aquáticas são usualmente prescritas para essa população, pois podem proporcionar à gestante um melhor condicionamento físico aeróbio e fortalecimento muscular. Em geral ocorre a liberação para a prática após o terceiro mês de gestação, com a autorização do obstetra (Jovanovic-Peterson \& Peterson, 1996).

A prática do exercício físico no meio aquático bem orientado proporciona: alívio de dores, relaxamento muscular, mantém e aumenta a amplitude de movimentos das articulações, reeduca músculos semiatrofiados ou atrofiados, desenvolve a força e a resistência, proporciona maior fortalecimento muscular, melhora ou reabilita as atividades funcionais da marcha, condicionamento cardiorrespiratório, controle do peso corporal, e ainda evitam tensões e desgaste das articulações devido à flutuabilidade, as condições da pele e da circulação também são melhoradas (Alves, 2009).

Os exercícios de alongamentos aquáticos reduzem a formação de edemas, muito comuns em gestantes, diminuem o estresse articular, diminuem os desconfortos musculares e melhoram a termo regulação, possibilitando ao feto uma estabilidade maior quanto à elevação de temperatura, propiciando o bem estar físico e mental já que durante a gestação ocorrem diversas alterações devido às mudanças hormonais, físicas e psicológicas, e por ser uma prática geralmente realizada em grupo, acaba se tornando algo prazeroso gerando uma socialização, diminuindo assim os riscos de depressão gestacional (Ferreira \& Alves, 2010).

Os exercícios para gestantes devem incluir a combinação de atividades aeróbias envolvendo grandes grupamentos musculares e atividades que desenvolvem força de determinados músculos. Acredita-se que uma musculatura abdominal forte possa ajudar no processo de expulsão do bebê. A força muscular de membros superiores também é muito importante para carregar a criança, que aumenta cada vez mais o seu peso (Botelho \& Miranda, 2012).

Durante uma gestação normal, mulheres praticantes de exercícios podem continuar a fazê-lo, com uma adequada prescrição para cada período gestacional para aprimorar os componentes da aptidão física (Oliveira et al., 2010).

A hidroginástica tem sido indicada como uma atividade adequada, pois tem como característica o baixo impacto articular, o aumento do retorno venoso devido à pressão hidrostática e comportamento de FC e PA mais baixos, evitando ainda a posição supina, que depois do primeiro trimestre pode resultar numa relativa obstrução do retorno venoso (Finkelstein et al., 2006). Nesse contexto, sua prática regular pode auxiliar na prevenção do aumento da pressão arterial (PA) durante a gestação, já que mulheres fisicamente ativas apresentam redução do risco de desenvolver hipertensão gestacional em aproximadamente 35\% (Sorensen et al., 2003).

Já está bem consolidado na literatura que a frequência cardíaca (FC) de repouso sofre influência com a imersão no meio líquido. Entretanto, os estudos que analisaram o comportamento da FC em meio líquido apresentam dados bastante controversos, sendo encontrados valores de bradicardia, taquicardia ou nenhuma alteração. Essa variação da FC de repouso no meio líquido depende da temperatura da água, da posição corporal, da profundidade de imersão, da FC inicial e da redução do peso hidrostático (Alberton \& Kruel, 2009).

Ao concordar que a atividade aquática é muito prescrita para as gestantes devido seus grandes benefícios para a mãe e bebê, o presente estudo tem como objetivo avaliar as adaptações fisiológicas ao exercício aquático em uma gestante hipertensa e uma gestante normotensa.

\section{Método}

\section{Participantes}

Estudo experimental do tipo relato de caso/controle composto por 2 mulheres, sendo 1 gestante hipertensa sedentária e controlada que faz uso de Aldomet (Metildopa) $500 \mathrm{mg}$ (3 vezes ao dia) e 1 gestante normotensa fisicamente ativa, ambas no terceiro trimestre de gestação. As características das participantes são apresentadas na tabela 1 a seguir.

Tabela 1. Características das participantes do estudo.

\begin{tabular}{lcc}
\hline Varivável & GNT & GH \\
\hline Idade (anos) & 31 & 35 \\
MCT (Kg) & 102,1 & 97,8 \\
Estatura (m) & 1,60 & 1,73 \\
\hline Nota: GNT: Grávida Normotensa; GH = Grávida Hipertensa; MCT = Massa Corporal
\end{tabular}

Nota: GNT: Grávida Normotensa; GH = Grávida Hipertensa; MCT = Massa Corporal Total.

\section{Procedimentos}

Os procedimentos para a realização do estudo foram executados de acordo com as normas éticas previstas na Resolução do Conselho Nacional de Saúde do Brasil № 466, de 12 de dezembro de 2012, que estabelece as diretrizes e normas regulamentadoras de pesquisas envolvendo seres humanos.

As participantes foram submetidas à avaliação das seguintes variáveis: frequência cardíaca $(F C)$, pressão arterial sistólica (PAS), pressão arterial diastólica (PAD), duplo produto (DP), variabilidade da frequência cardíaca (VFC), e glicose sanguínea (GLI). As variáveis hemodinâmicas foram avaliadas por meio de um frequencímetro da marca Polar ${ }^{\circledast}$ (modelo RS800CX), um esfigmomanômetro eletrônico da marca OMRON ${ }^{\circledR}$ (modelo HEM-7320). O valor do DP foi obtido por meio do produto entre a PAS e a FC (V DBH, 2007). A GLI foi avaliada por meio do monitor de glicemia da marca Roche ${ }^{\circledR}$ (modelo Accutrend Plus).

As variáveis dependentes foram avaliadas em quatro momentos distintos: em decúbito dorsal após repouso de 15’ (M1), em imersão em piscina com água na altura do manúbrio esternal após repouso de $5^{\prime}$ (M2), imediatamente após a sessão de exercício aquático (M3), e em decúbito dorsal após repouso de $5^{\prime}$ (M4) respectivamente, exceto para a GLI que foi aferida apenas em M1, M3 e M4.

A sessão de exercício aquático consistiu em movimentos de adução e abdução horizontal de ombros combinados com flexão e extensão de quadril sem a utilização de implementos. Todos os movimentos foram realizados em posição em pé, em piscina com temperatura em $31^{\circ} \mathrm{Ce}$ $\mathrm{pH}$ de 7,8 , com água na altura do manúbrio esternal. Durante a sessão de exercícios, as participantes permaneceram em zona alvo de treinamento compreendida entre $60 \%$ e $80 \%$ da frequência cardíaca máxima. 
Para evitar que o fenômeno de hemoconcentração pudesse interferir nos valores das variáveis analisadas, durante a sessão de exercício aquático as voluntárias fizeram consumo de água ad libitum.

\section{Análise estatística}

Foi realizada a análise descritiva dos dados. Para avaliação de alterações nas variáveis dependentes do estudo, calculou-se a variação percentual dos dados

\section{Resultados}

Os valores obtidos para as variáveis dependentes nos distintos momentos do estudo são apresentados na tabela 2 a seguir.

Tabela 2. Variáveis fisiológicas das gestantes.

\begin{tabular}{ccccccccc}
\hline & \multicolumn{3}{c}{ M1 } & \multicolumn{2}{c}{ M2 } & \multicolumn{2}{c}{ M3 } & \multicolumn{2}{c}{ M4 } \\
\hline & GNT & GH & GNT & GH & GNT & GH & GNT & GH \\
\hline FC & 91 & 100 & 95 & 84 & 134 & 127 & 95 & 93 \\
PAS & 122 & 144 & 111 & 127 & 126 & 146 & 124 & 129 \\
PAD & 71 & 86 & 50 & 65 & 43 & 71 & 63 & 81 \\
DP & 11102 & 14100 & 10545 & 10668 & 17286 & 18542 & 11780 & 11997 \\
VFC & 17,4 & 20,3 & 17,2 & 25,6 & 6,7 & 6,1 & 18,4 & 24,7 \\
GLI & 138 & 125 & - & - & 85 & 81 & 80 & 79
\end{tabular}

Nota: GNT = Grávida Normotensa; GH = Grávida Hipertensa; FC = frequência cardíaca $(\mathrm{bpm}) ; \mathrm{PAS}=$ Pressão arterial sistólica $(\mathrm{mmHg}) ; \mathrm{PAD}=$ Pressão arterial diastólica $(\mathrm{mmHg}) ; \mathrm{DP}=$ Duplo produto; VFC $=$ Variabilidade da Frequência Cardíaca valor absoluto; $\mathrm{GLI}=$ glicose.

\section{Discussão}

É comum observar alterações hemodinâmicas maternas. Tais mudanças incluem o aumento de volume sanguíneo, aumento de frequência cardíaca ( $F C$ ), do volume sistólico, do débito cardíaco (DC) e diminuição de resistência vascular sistêmica e da pressão arterial (PA). Na tabela 2, estão descritas respostas fisiológicas e metabólicas agudas ao exercício em meio líquido.

Podemos notar que as variáveis de FC não sofreram alterações significantes quando comparados os momentos M1 e M4. A FC dos dois casos não apresentou alterações relativamente alta nas respectivas condições, o que leva ao entendimento de que, durante a gravidez, ocorrem adaptações que são necessárias para maior oferta de nutrientes e oxigênio ao feto durante o repouso e atividade física (Artal, O'Toole, White, 2003), contudo, na GH é possível verificar uma diminuição da $F C_{\text {Rep }}$ quando comparados $M 1$ e M4. Já está bem consolidado na literatura que uma $\mathrm{FC}$ de repouso baixa tende a representar uma melhora no quadro de saúde, enquanto valores mais altos aparentemente estão relacionados a risco aumentado de mortalidade (La Rovere et al., 2002). Afim de sustentar esse racional fisiológico, um dos fenômenos que explica a diminuição da $F_{\text {Rep }}$ é o aumento do tônus vagal e consequentemente uma bradicardiaca (Artal, O'Toole, White, 2003). No meio líquido, os dados apontam para a ocorrência da diminuição da FC durante e pós imersão. Isso ocorre devido a uma bradicardia induzida pela imersão corporal, o que explica essa resposta aguda da FC é o aumento da pressão hidrostática sobre os indivíduos.

A respeito das respostas da PAS da GN foi possível observar que não houve alterações significativas, entretanto, na $\mathrm{GH}$, houve uma diminuição relevante da PAS quando comparados os momentos $\mathrm{M} 1$ e M4 ( $\Delta \% 10,4 \mathrm{mmHg})$, essa redução da PAS foi sustentada, caracterizando um efeito hipotensor pós exercício (HPE). A HPE caracteriza-se pela redução da PA durante o período de recuperação (Brum, Forjaz, Tinucci, \& Negrão, 2004), fazendo com que valores pressóricos observados pós-exercícios permaneçam inferiores aqueles que antecederam o esforço. Acredita-se que esse fenômeno esteja associado a maior perfusão sanguínea miocárdica. Corroborando com essas afirmativas, estudos já demonstraram que uma única sessão de exercício é capaz de reduzir a PA de indivíduos hipertensos (Finkelsein et al., 2009). Além disso, a imersão na água em temperatura neutra (30 a $32^{\circ} \mathrm{C}$ ) faz com que ocorra redução na resistência periférica pela dilatação das arteríolas, levando a uma queda da pressão arterial. Os efeitos da gravidade são reduzidos embaixo da água, o que desvia sangue e líquido dos membros inferiores para o tórax, fazendo com que esse aumento do retorno venoso leve à estimulação dos barorreceptores, e essa combinação de eventos determina redução da pressão arterial (Negrão \& Rondon, 2001). Houve decréscimo dos valores da PAD nas duas gestantes, isso se dá por conta de um consequente aumento dos vasos sanguíneos uterinos, da circulação útero-placentária e diminuição da resistência vascular da pele e rins (Brum, Forjaz, Tinucci, \& Negrão, 2004).

A glicose apresentou redução significativa quando comparada aos valores basais $\mathrm{GN}(\Delta \% 42,02), \mathrm{GH}(\Delta \% 36,8)$. Estudos anteriores relataram aumento da glicose no período gestacional (Dye et al., 1997), face ao exposto, um decréscimo na glicose representa um fator importante na proteção materna e na saúde do feto.

\section{Conclusões}

A partir dos resultados apresentados é possível sugerir o exercício físico no meio aquático para gestantes hipertensas, uma vez que após a prática ocorreu decréscimo de valores fisiológicos basais como pressão arterial e frequência cardíaca, não trazendo nenhum risco ao feto, além de atuar como um fator preventivo a diabetes gestacional, auxiliando na melhora cardiorrespiratória e aumento do retorno venoso.

Um fator limitante que deve ser observado é o acompanhamento nutricional para que não ocorra picos hipoglicêmicos durante a atividade física influenciando negativamente o desempenho da atividade e trazendo mal-estar a gestante praticante e a ausência de um follow-up.

\section{Contribuição e implicações práticas}

O presente estudo oferece subsídios teóricos para a prescrição do exercício aquático para gestantes, levando em consideração a metodologia apresentada, para no que diz respeito o comportamento das variáveis fisiológicas e metabólicas.

\section{Agradecimentos}

Os autores agradecem às gestantes voluntárias para o desenvolvimento do presente estudo.

\section{Referências}

Alberton, C., \& Kruel, L. F. (2009). Influência da imersão nas respostas cardiorrespiratórias em repouso. Revista Brasileira de Medicina do Esporte, 15(3), 228-232.

Alves, M. V. P. (2009). Hidroginástica: Novas Abordagens. Rio de Janeiro: Atheneu.

Artal, R., \& Gardin, S. K. (1999). Perspectiva histórica. In R. Artal, A. R. Wiswell, L. R. Drinkwater (Eds.), O exercício na gravidez. São Paulo: Manole.

Artal R., O'toole, M., \& White, S. (2003). Guideline of the American College of Obstetrician and Gynecologists for exercice during pregnancy and postpartun period. Britsh Journal of Sports Medicine, 37(1), 6-12. 
Botelho, P. R., \& Miranda, E. F. (2012). Principais recomendações sobre a prática de exercícios físicos durante a gestação. Revista Cereus, 6, 1-10.

Brum, P., Forjaz, C., Tinucci, T., \& Negrão, C. (2004). Adaptações agudas e crônicas do exercício físico no sistema cardiovascular. Revista Paulista Educação Física, 18, 21-31.

Dye, T. D., Knox, K. L., Artal, R., Aubry, R. H., \& Wojtowycz, M. A. (1997). Physical activity, obesity, and diabetes in pregnancy. American Journal Epidemiology, 146(11), 961-965.

Ferreira, C., \& Alves, M. C. (2010). Principais mudanças fisiológicas na gestação e exercício. EF Deportes Revista Digital, 15(147),1.

Finkelsein I., Bgeginski R., Figueiredo, P. A. P., Alberton, C. L., Stein, R., \& Kruel, L. F. M. (2009). Comparação das respostas pressóricas e do consumo de oxigênio de gestantes e não gestantes, em exercício contínuo e progressivo, nos meios terra e água. Revista Brasileira de Medicina, 66, 174-177.

Finkelstein, I., Bgeginski, R., Tartaruga, M. P., Alberton, C. L., \& Kruel, L. F. (2006). Comportamento da frequência cardíaca e da pressão arterial, ao longo da gestação, com treinamento no meio líquido. Revista Brasileira de Medicina do Esporte, 12(6), 376-380.

Haskell, W. L., Lee, I. M., Pate, R. R., Powell, K. E., Blair, S. N., Franklin, B. A., et al. (2007). Physical activity and public health: updated recommendation for adults from the American College of Sports Medicine and the American Heart Association. Medicine \& Science in Sports \& Exercise, 39(8), 1423-1434.

Jovanovic-Peterson L., \& Peterson C. M. (1996). Exercise and the nutritional management of diabetes during pregnancy. Obstetrics and Gynecology Clinics of North America, 23(1), 75-86.

Kruel, L. F. M., Moraes, E. Z. C., \& Ávila, A. O. V., \& Sampedro, R. M. F. (2001). Alterações fisiológicas e biomecânicas em indivíduos praticando exercícios de hidroginástica dentro e fora d'água. Revista Kinesis, 104-129.

La Rovere, M. T., Bigger, J. T., Jr, Marcus, F. I., Mortara, A., \& Schwartz, P. J. (1998). Baroreflex sensitivity and heart-rate variability in prediction of total cardiac mortality after myocardial infarction. ATRAMI (Autonomic Tone and Reflexes After Myocardial Infarction) Investigators. Lancet, 351, 478-484.

Negrão, C. E., \& Rondon, M. U. P. B. (2001). Exercício físico, hipertensão e controle barorreflexo da pressão arterial. Revista Brasileira de Hipertensão, 8(1), 89-95.

Oliveira L., Vasconcelos M., Carvalho S., \& Gadelha M. (2010). Repercussões da imersão sobre a pressão arterial em gestantes. Revista Brasileira de Ciências do Esporte, 32(2), 245-258.

SBEM. Sociedade Brasileira de Endocrinologia e Metabologia (2008) Diabetes Mellitus Gestacional. Revista da Associação Medica Brasileira, 54(6), 471-486.

Sorensen, T. K., Williams, M. A., Lee, I. M., Dashow, E. E., Thompson, M. L., \& Luthy, D. A. (2003). Recreational Physical Activity During Pregnancy and Risk of Preeclampsia. Hypertension, 41(6), 12731280.

Thomas, J. R., Nelson, J. K., \& Silverman, S. J. (2008). Métodos de pesquisa em atividade física. 5ed. São Paulo: Artmed.

SBH (2009). V Diretrizes Brasileiras de Hipertensão Arterial. Arquivos Brasileiros de Cardiologia, 89(3), e24-e79. 